\section{Desigualdades no comprometimento da renda domiciliar dos brasileiros com gastos privados em assistência odontológica}

\author{
Inequalities in Brazilian families' income \\ commitment to out-of-pocket spending \\ on dental care
}

Andreia Morales Cascaes 1

Aryane Marques Menegaz 1

Andressa Raquel Spohr 1

Rafael Aiello Bomfim 2

Aluísio J. D. Barros 1

doi: 10.1590/0102-311X00104017

\title{
Resumo
}

O objetivo foi investigar as desigualdades no comprometimento da renda domiciliar com gastos privados em assistência odontológica no Brasil. Foram analisados dados de 55.970 domicílios brasileiros que participaram da Pesquisa de Orçamentos Familiares, de abrangência nacional, no período de 2008-2009. O comprometimento dos gastos privados com assistência odontológica na renda familiar foi calculado pela divisão do gasto médio domiciliar per capita anual pela renda familiar per capita anual e estimado segundo quatro categorias: $>0 \%, \geq 5 \%, \geq 10 \% e \geq 20 \%$. A análise do comprometimento na renda foi realizada apenas para os domicílios com gasto positivo. Apenas 2.961 (7\%) dos domicílios reportaram gastos positivos com assistência em odontologia. No geral, o gasto médio per capita anual foi de $R \$ 42,19 e$, de $R \$$ 602,47 entre aqueles com gasto positivo. Os domicílios que possuem os maiores gastos absolutos com assistência odontológica são aquelas pertencentes à área urbana e ao quinto mais rico. Em contrapartida, os domicílios com maior comprometimento na renda pertencem à área rural e ao quinto mais pobre. Entre os que informaram gasto positivo, 55\% dos domicílios do quinto mais pobre comprometeram $\geq 20 \%$ de sua renda com assistência odontológica. $O$ percentual é de apenas 6\% no grupo mais rico da população. Os domicílios mais pobres das regiões mais ricas (Centro-oeste, Sul e Sudeste) apresentaram os maiores comprometimentos de renda. As desigualdades socioeconômicas nos gastos e no comprometimento de renda com assistência odontológica são evidentes. A avaliação dessas desigualdades torna-se relevante para avaliação e orientação de políticas públicas em saúde.

Economia em Odontologia; Renda; Gastos em Saúde

\author{
Correspondência \\ A. M. Cascaes \\ Universidade Federal de Pelotas. \\ Rua Gonçalves Chaves 457, Pelotas, RS 96015-560, Brasil. \\ andreia.cascaes@ufpel.edu.br \\ 1 Universidade Federal de Pelotas, Pelotas, Brasil. \\ 2 Universidade Federal do Mato Grosso do Sul, Campo Grande, \\ Brasil.
}




\section{Introdução}

O Brasil é o único país do mundo, ao menos do ponto de vista legal, com sistema de saúde público universal em que o gasto privado ultrapassa o gasto estatal ${ }^{1}$. Em 2009, a proporção do gasto público em relação ao gasto total em saúde (público mais privado) no Brasil pode ser considerada baixa $(43,6 \%) \mathrm{em}$ relação a outros países com sistema universal de saúde, como Canadá (70,6\%), França (77,9\%), Nova Zelândia (83\%) e Inglaterra (84,1\%) 2. Em 2013, dados brasileiros da Conta-Satélite de Saúde assinalam um gasto total em saúde de R 421 milhões, sendo R 231 milhões (4,4\% do PIB) provenientes das famílias e do setor privado e R\$ 190 milhões do setor público (3,6\% do PIB) 3. O gasto privado em saúde, especialmente não programado, pode produzir gastos catastróficos no orçamento familiar e ocasionar impactos negativos na população. Muitas famílias, particularmente aquelas com menores condições socioeconômicas, cortam despesas importantes para sua subsistência a fim de conseguir acessar o serviço de saúde ou aderir a tratamentos 1,4,5.

Informações sobre os gastos privados com saúde das famílias brasileiras podem ser obtidas pelas Pesquisas de Orçamentos Familiares (POF), realizadas periodicamente, desde 1987, pelo Instituto Brasileiro de Geografia e Estatística (IBGE) e também do componente de saúde das Pesquisas Nacionais por Amostra de Domicílios (PNAD) de 1998, 2003 e 2008. Diversos trabalhos publicados valendo-se dessas bases de dados têm como foco a análise do perfil do gasto das famílias e a questão distributiva a ele relacionada $6,7,8,9,10$. Tais estudos apontam que a renda e escolaridade são fator determinantes dos gastos privados com saúde, ou seja, quanto maior a renda e maior a escolaridade, maior o gasto absoluto com saúde. Por outro lado, são as famílias mais pobres que comprometem, proporcionalmente, grande parte de sua renda com esses dispêndios 10,11.

No que se refere à assistência odontológica, poucos estudos brasileiros incluíram os gastos privados com esse setor em suas análises. Garcia et al. 6 identificaram que as despesas com tratamento dentário das famílias residentes nas regiões metropolitanas brasileiras ocupam a terceira posição do total de gastos com saúde e que os valores despendidos vêm reduzindo ao longo das três últimas POF, especialmente entre as famílias de menor renda, uma vez que entre aquelas com maior renda, os valores permaneceram estáveis. Boing et al. 5 identificaram que os gastos com odontologia contribuíram em $5 \%$ e $2 \%$ com o empobrecimento das famílias brasileiras decorrente dos gastos em saúde nos anos de 2002-2003 e 2008-2009, consecutivamente. Cascaes et al. ${ }^{9}$ quantificaram e descreveram as desigualdades no padrão dos gastos per capita com assistência odontológica avaliados na POF 20082009. Os maiores gastos foram encontrados nos domicílios das regiões Sudeste e Sul, cujos chefes apresentavam cor da pele branca, maior escolaridade e renda e naqueles com presença de indivíduos abaixo de 60 anos de idade.

Embora o panorama distributivo dos gastos privados odontológicos tenha sido descrito na literatura, até o momento, nenhuma revisão sistemática ou artigo publicado avaliou e discutiu em detalhes o impacto dos gastos privados em odontologia na renda familiar dos brasileiros. Os serviços privados pagos pelos usuários ainda são o principal meio de acesso à assistência odontológica no Brasil, o Sistema Único de Saúde (SUS) ocupa a segunda posição 12. A alta prevalência de doenças bucais na população brasileira 13 , aliada às dificuldades no acesso aos serviços públicos odontológicos, sobretudo aos especializados, geram demandas e, consequentemente, gastos privados com o setor. O quanto esse tipo de gasto compromete o orçamento dos brasileiros é uma informação pouco conhecida, podendo auxiliar no planejamento de serviços odontológicos no país e contribuir para identificar segmentos que requerem maior atenção de políticas públicas de saúde e proteção social.

Tendo em vista a disponibilidade de dados em nível nacional sobre os gastos privados com assistência odontológica, a relevância da análise do comprometimento da renda das famílias com esse tipo de gasto e a escassez de publicações sobre o tema, o presente estudo se propôs a analisar as desigualdades no comprometimento da renda das famílias com gastos privados em assistência odontológica no Brasil. 


\section{Metodologia}

Trata-se de um estudo transversal que analisou dados oriundos da POF, de abrangência nacional, conduzidas nas áreas urbana e rural do Brasil pelo IBGE, no período de 19 de maio de 2008 a 18 de maio de 200914.

Por meio das POF, é possível conhecer o perfil das condições das famílias mediante análise de seus orçamentos domésticos. Essas pesquisas trazem informações acerca dos tipos de gastos, valor, quantidade, especialidade de serviços, bem como os rendimentos das famílias. Os gastos com saúde nas POF são captados segundo categorias distintas, e uma dessas categorias é a assistência em odontologia.

Nos anos 2008 e 2009, a POF investigou 55.970 domicílios brasileiros 14. Os domicílios foram selecionados por um plano de amostragem por conglomerados com sorteio dos setores censitários, em primeiro estágio, e de domicílios, em segundo. Os setores censitários foram selecionados com probabilidade proporcional ao número de domicílios existentes em cada setor, dentro de cada estrato amostral, considerados unidades primárias no planejamento amostral das pesquisas domiciliares conduzidas pelo IBGE. Foram agrupados previamente considerando a localização geográfica do setor (região, estado, situação urbana ou rural e municípios da capital, da região metropolitana e demais municípios) e, internamente, em cada lócus geográfico, a variação socioeconômica das famílias residentes, pela renda do indivíduo responsável pelo domicilio. No segundo estágio de seleção, foram incluídos os domicílios particulares permanentes, por amostragem aleatória simples sem reposição, em cada estrato amostral, considerados unidades secundárias de amostragem, dentro de cada setor censitário.

Os setores censitários foram distribuídos ao longo dos quatro trimestres da pesquisa, garantindo que em todos os trimestres os estratos geográficos e socioeconômicos estivessem representados pelos domicílios selecionados. Dos 4.696 setores selecionados, dois apresentaram todas as entrevistas como não realizadas e, portanto, foram excluídos da amostra analisada no presente estudo. Pela amostra da POF 2008-2009, pode-se fazer inferência para as cinco regiões (Norte, Nordeste, Sul, Sudeste e Centro-oeste), as 27 Unidades da Federação, as nove regiões metropolitanas e as capitais dos estados em relação à zona urbana.

A coleta de dados da POF 2008-2009 foi realizada por agentes de pesquisa por intermédio de entrevista domiciliar, utilizando meios eletrônicos para a obtenção das informações. A POF agrega o conjunto de informações de seis questionários: domicílios, despesa coletiva, caderneta de despesa coletiva, despesa individual, rendimento individual e avaliação das condições de vida.

Particularmente, os gastos referentes à assistência em odontologia - o que interessa ao presente estudo - foram obtidos no bloco de despesas individuais, os quais somam as despesas com planos exclusivamente odontológicos (empresarial e privado), consulta odontológica e tratamentos dentários. Os tratamentos dentários somaram gastos com obturação dentária, extração dentária, prótese e aparelho dentário, aparelho ortodôntico, pivô, dentadura, tratamento dentário, tratamento de canal dentário, colocação de jaqueta dentária, manutenção de aparelho dentário, manutenção de aparelho ortodôntico e implante de dente. O período recordatório relativo aos gastos com assistência odontológica se refere a noventa dias antes da entrevista.

Os gastos com assistência odontológica foram estimados levando em conta o tamanho do domicílio. Para tanto, dividiu-se o gasto anual total pelo número de moradores do domicílio, obtendo-se o gasto médio domiciliar per capita anual.

O comprometimento dos gastos com assistência odontológica na renda familiar foi calculado pela divisão do gasto médio domiciliar per capita anual pela renda familiar per capita anual. Os valores foram multiplicados por 100 para expressar as porcentagens, conforme recomendado pelo IBGE para o cálculo do indicador E.9.114. Em seguida, o comprometimento do gasto com assistência odontológica na renda familiar foi estimado segundo quatro categorias, previamente utilizadas na literatura: $>0 \%, \geq 5 \%, \geq 10 \%$ e $\geq 20 \% 10,11$.

Os dados da POF 2008-2009 foram obtidos no sítio eletrônico do IBGE (http://www.ibege.gov. br) e são de domínio público. O programa estatístico Stata versão 14.0 (StataCorp LP, College Station, Estados Unidos) foi utilizado para organizar o banco e analisar os dados. As análises consideraram o desenho amostral da POF e os fatores de expansão disponibilizados pelo IBGE. Os valores apresentados foram deflacionados, utilizando como referência a data de 15 de janeiro de 2009, conforme recomendação do IBGE. 
O montante de gastos absolutos médios domiciliares per capita com assistência odontológica e o comprometimento médio na renda com tais gastos foram calculados e descritos para as macrorregiões, estados da federação, zona urbana e rural, destacando as desigualdades segundo quintos de renda familiar per capita anual. As diferentes categorias de comprometimento percentual do gasto com odontologia na renda das famílias foram descritas segundo quintos de renda. Cinco domicílios com renda familiar anual igual a zero foram excluídos. Somente os domicílios com gasto positivo foram incluídos na análise de comprometimento de renda, uma vez que a imensa maioria dos domicílios não relatou gasto com odontologia. Além disso, o interesse principal desta pesquisa é conhecer o comprometimento do orçamento daqueles que informaram gastos.

\section{Resultados}

Do total de domicílios investigados na POF 2008-2009, apenas 2.961 (7\%) reportaram gastos com assistência odontológica. Entre estes, o percentual médio de domicílios com renda comprometida com assistência odontológica foi de 13,3\% (IC95\%: 9,8; 16,9). O percentual de domicílio que comprometeram $\geq 5 \%$, $\geq 10 \%$ e $\geq 20 \%$, respectivamente, foi de 51,3\% (IC95\%: 48,3; 54,3), 32,1\% (IC95\%: 29,7; 34,6 ) e $14,5 \%$ (IC95\%: 12,8; 16,5).

A média per capita de gastos no geral foi de $\mathrm{R} \$ 42,19$ e a média per capita de gasto somente de quem relatou gasto foi em torno de $\mathrm{R} \$ 602,47$. Os valores per capita dos gastos aumentaram conforme a renda domiciliar (Tabela 1). No quinto mais rico, a média de gasto per capita geral foi de $\mathrm{R} \$ 151,40$ e a média de gasto per capita positivo foi de $\mathrm{R} \$ 1.077,73$. Já no quinto mais pobre, esses valores somaram, respectivamente, $\mathrm{R} \$ 3,24$ e $\mathrm{R} \$ 161,56$ (Tabela 1).

A Tabela 1 apresenta também a proporção de renda familiar per capita comprometida em valores iguais ou superiores a $0 \%, 5 \%, 10 \%$ e $20 \%$ com gastos em assistência odontológica, entre aqueles com gasto positivo. $\mathrm{O}$ comprometimento de renda com assistência odontológica foi expressivamente maior em todas as categorias, entre os mais pobres (Q1) quando comparados com os mais ricos (Q5). Considerando o comprometimento de $10 \%$ ou mais de renda per capita, 14,7\% dos mais ricos (Q5) foram afetados, enquanto, entre os mais pobres, o comprometimento chegou a alcançar 72,2\%. Quando se considerou comprometimento igual ou maior a 20\%, 54,1\% dos indivíduos mais pobres (Q1) estavam nessa condição, contra 5,9\% dos mais ricos (Q5).

Tabela 1

Distribuição dos gastos médios domiciliares per capita (em Reais) e proporção de renda familiar per capita comprometida com gastos em assistência odontológica. Pesquisa de Orçamentos Familiares (POF), Brasil, 2008-2009.

\begin{tabular}{|c|c|c|c|c|c|}
\hline & \multicolumn{5}{|c|}{ Quintos de renda familiar per capita } \\
\hline & Q1 (mais pobres) & Q2 & Q3 & Q4 & Q5 (mais ricos) \\
\hline Gasto per capita no geral (R\$) [média (IC95\%)] & $\begin{array}{c}3,24 \\
(2,42 ; 4,06)\end{array}$ & $\begin{array}{c}6,99 \\
(5,83 ; 8,15)\end{array}$ & $\begin{array}{c}17,81 \\
(13,90 ; 21,71)\end{array}$ & $\begin{array}{c}31,50 \\
(26,57 ; 36,45)\end{array}$ & $\begin{array}{c}151,40 \\
(88,31 ; 214,50)\end{array}$ \\
\hline Gasto per capita positivo (R\$) * [média (IC95\%)] & $\begin{array}{c}161,56 \\
(131,73 ; 191,39)\end{array}$ & $\begin{array}{c}185,54 \\
(164,85 ; 206,24)\end{array}$ & $\begin{array}{c}282,91 \\
(228,79 ; 337,03)\end{array}$ & $\begin{array}{c}354,22 \\
(308,84 ; 399,59)\end{array}$ & $\begin{array}{c}1.077,73 \\
(629,30 ; 1.526,15)\end{array}$ \\
\hline \multicolumn{6}{|l|}{ Comprometimento na renda * [\% (IC95\%)] } \\
\hline$>0 \%$ & $\begin{array}{c}69,12 \\
(13,96 ; 124,27)\end{array}$ & $\begin{array}{c}16,53 \\
(14,77 ; 18,29)\end{array}$ & $\begin{array}{c}13,07 \\
(11,04 ; 15,11)\end{array}$ & $\begin{array}{c}9,60 \\
(8,46 ; 10,74)\end{array}$ & $\begin{array}{c}6,72 \\
(4,94 ; 8,51)\end{array}$ \\
\hline$\geq 5 \%$ & $\begin{array}{c}86,16 \\
(80,31 ; 90,45)\end{array}$ & $\begin{array}{c}75,41 \\
(69,76 ; 80,30)\end{array}$ & $\begin{array}{c}63,48 \\
(57,02 ; 69,49)\end{array}$ & $\begin{array}{c}54,15 \\
(48,99 ; 59,22)\end{array}$ & $\begin{array}{c}32,39 \\
(27,74 ; 37,42)\end{array}$ \\
\hline$\geq 10 \%$ & $\begin{array}{c}72,28 \\
(65,25 ; 79,32)\end{array}$ & $\begin{array}{c}57,48 \\
(51,27 ; 63,69)\end{array}$ & $\begin{array}{c}42,95 \\
(37,39 ; 48,52)\end{array}$ & $\begin{array}{c}31,66 \\
(26,75 ; 36,57)\end{array}$ & $\begin{array}{c}14,77 \\
(11,46 ; 18,08)\end{array}$ \\
\hline$\geq 20 \%$ & $\begin{array}{c}54,14 \\
(45,52 ; 62,78)\end{array}$ & $\begin{array}{c}30,44 \\
(24,57 ; 36,32)\end{array}$ & $\begin{array}{c}16,68 \\
(12,35 ; 20,99)\end{array}$ & $\begin{array}{c}10,55 \\
(7,43 ; 13,68)\end{array}$ & $\begin{array}{c}5,98 \\
(3,69 ; 8,28)\end{array}$ \\
\hline
\end{tabular}

* Somente em quem relatou gasto positivo. Valor de p do teste de tendência linear para todas as associações < 0,001. 
Ao analisar a distribuição dos gastos per capita positivos com assistência odontológica, a Região Centro-oeste (incluindo o Distrito Federal) é a que apresenta maior gasto per capita positivo (R\$ $1.632,53)$, seguida pelo Sudeste ( $\mathrm{R} \$ 1.207,10)$, Sul (R\$ 1.010,12), Norte (R\$ 583,09) e Nordeste (R\$ 468,28). A Figura 1 apresenta a média de gastos domiciliares per capita positivos (em Reais) e proporção média da renda familiar comprometida com assistência odontológica nas macrorregiões brasileiras e nas zonas urbana e rural, segundo quintos de renda familiar. Nas cinco regiões do país, as famílias pertencentes ao quinto mais rico são as que relataram maior gasto com serviços odontológicos. Entretanto, são as famílias dos quintos mais pobres (Q1 e Q2), em todas as regiões, que mais comprometem sua renda com os gastos em odontologia. Com relação à zona de residência, os domicílios da zona urbana pertencentes ao primeiro e ao quinto quinto de renda, gastam ligeiramente mais com serviços odontológicos do que aqueles que vivem na zona rural. Os domicílios dos quintos intermediários residentes em zona rural relataram um gasto superior ao dos da zona urbana. A análise do comprometimento da renda segue padrão anteriormente descrito, maior comprometimento no orçamento dos domicílios dos quintos mais pobres em relação aos ricos, em ambas as zonas de residência.

\section{Figura 1}

Média de gastos domiciliares per capita positivos (em Reais) e proporção média da renda familiar comprometida com assistência odontológica nas macrorregiões brasileiras e zona de residência, segundo quintos de renda familiar. Pesquisa de Orçamentos Familiares (POF), Brasil, $2008-2009$.

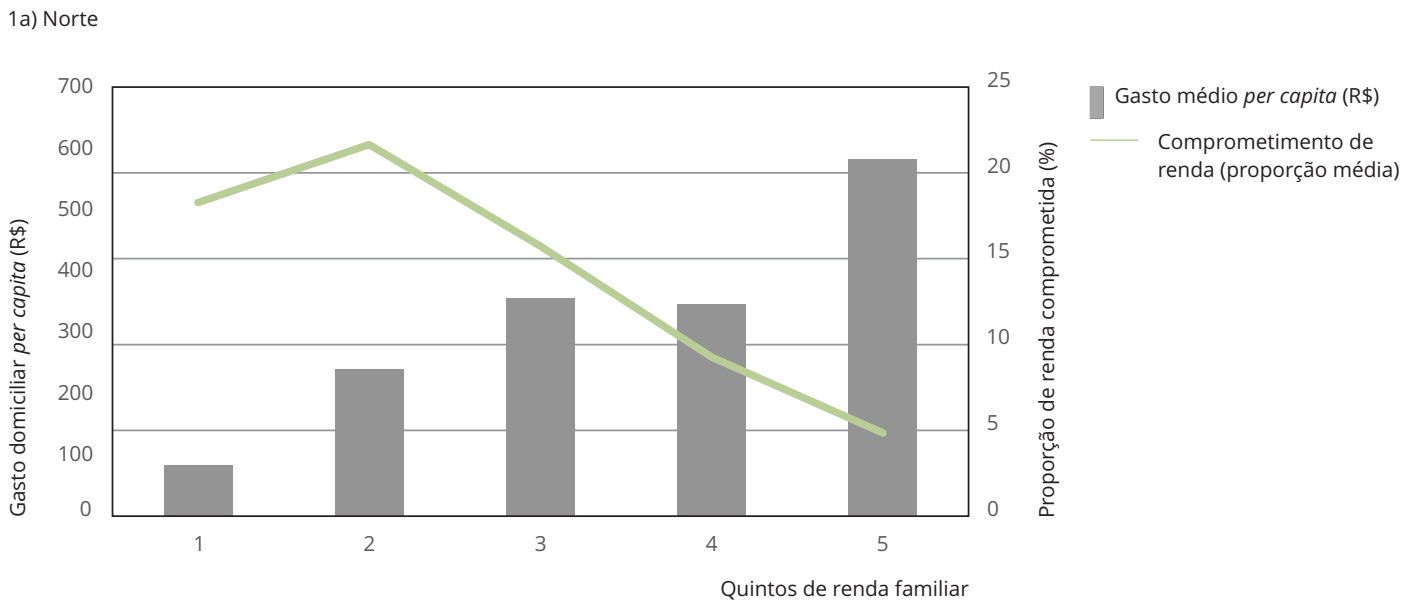

1b) Nordeste

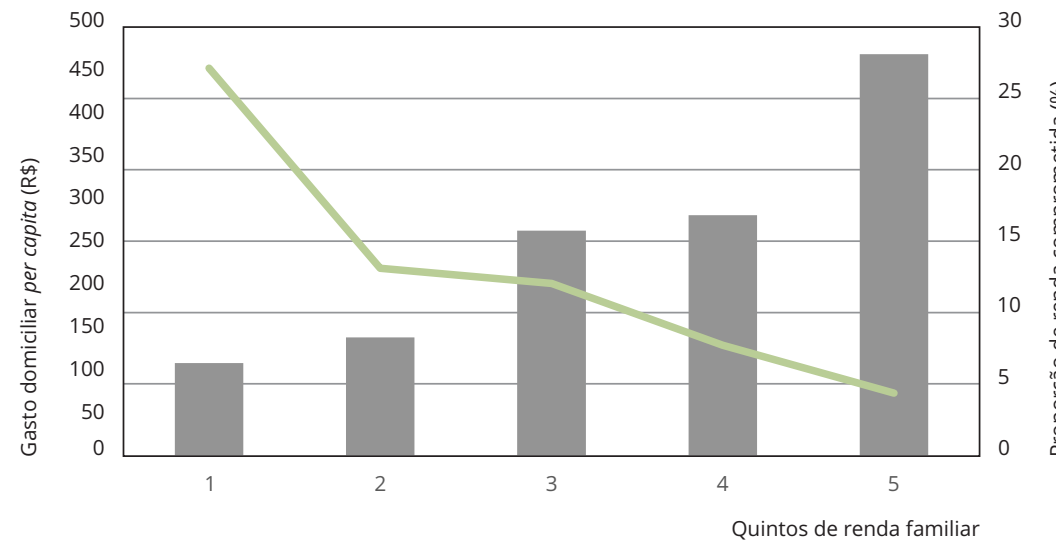

Gasto médio per capita $(\mathrm{R} \$)$

- Comprometimento de renda (proporção média)

(continua) 
Figura 1 (continuação)

1c) Centro-oeste

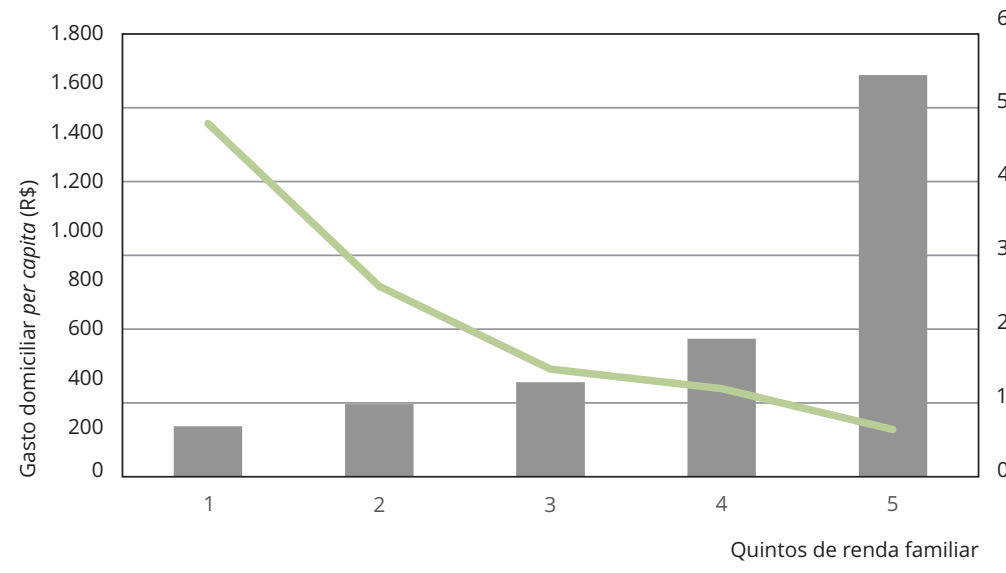

60

Gasto médio per capita (R\$)

50

- Comprometimento de renda (proporção média)

1d) Sudeste
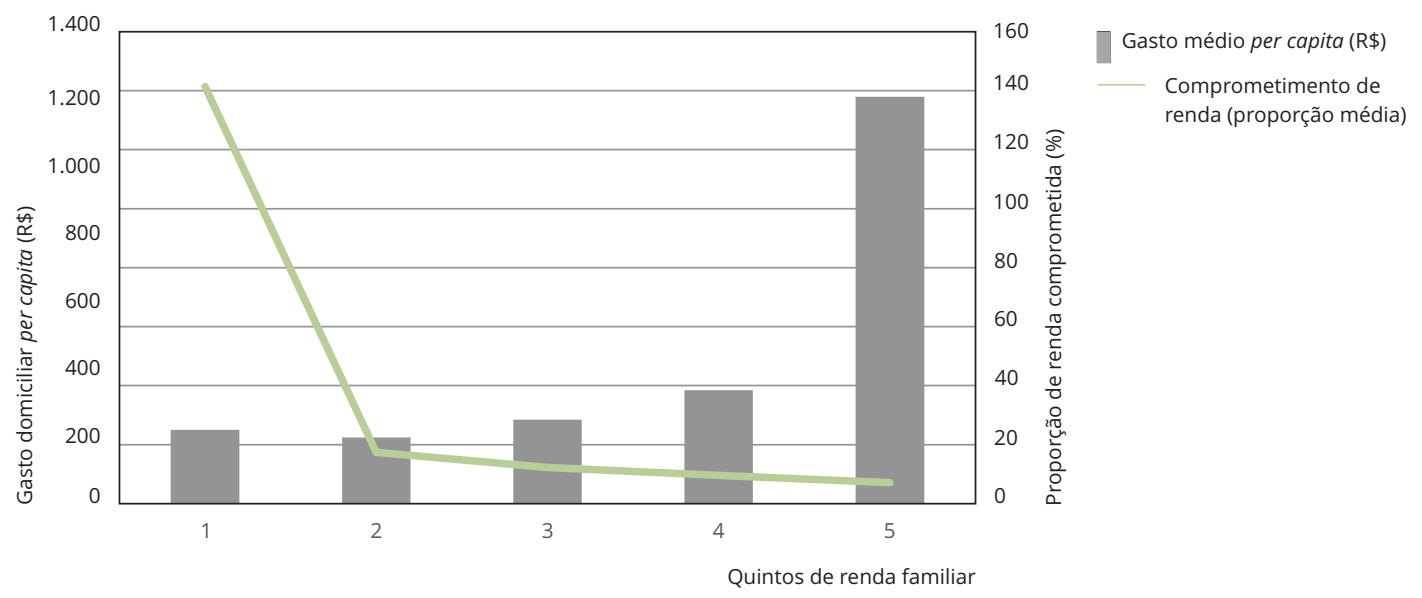

1e) Sul

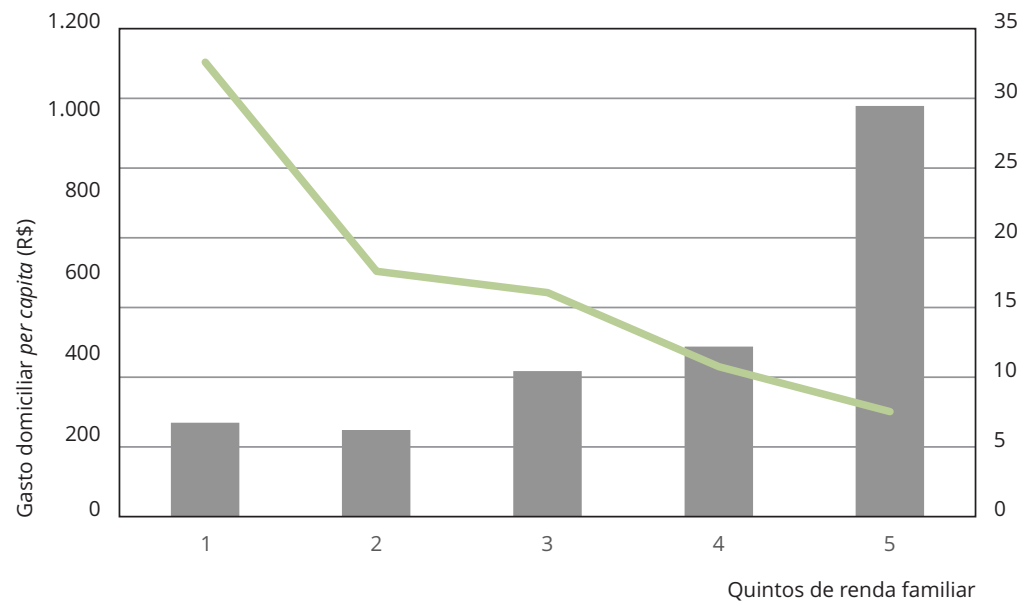

Gasto médio per capita (R\$)

Comprometimento de

renda (proporção média)

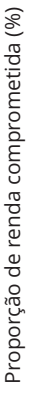

(continua) 


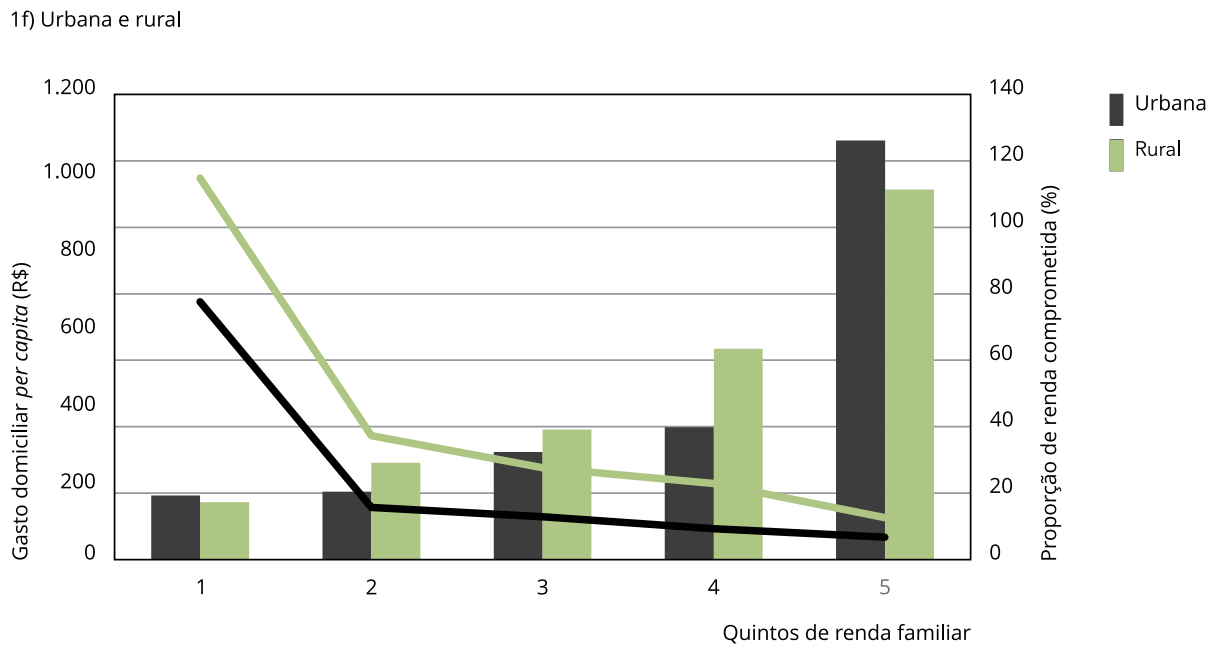

A Figura 2 descreve a média de gastos domiciliares per capita positivos (em Reais) e proporção média da renda familiar comprometida com assistência odontológica de acordo com os estados brasileiros. Os estados que apresentaram as maiores médias de gastos domiciliares per capita positivos foram Goiás (R\$ 1.041,34), Distrito Federal (R\$ 906,20) e Paraná (R\$ 849,21) e os de menor gasto foram o Rio Grande do Norte (R\$ 151,05), Amazonas (R\$ 162,23) e Pernambuco (R\$ 207,44). No entanto, o Estado de Espírito Santo foi o que apresentou a maior proporção média $(59,54 \%)$ de renda familiar comprometida com assistência odontológica, seguido do Rio de Janeiro (28,34\%) e Tocantins $(23,75 \%)$. Nos demais estados, a proporção média de comprometimento na renda domiciliar não chegou a 20\%, variando de 6,98\%, na Paraíba a 18,35\%, no Estado de Roraima.

\section{Discussão}

O presente estudo estimou os gastos das famílias brasileiras com serviços odontológicos e apresentou a magnitude das desigualdades no comprometimento da renda domiciliar com esses gastos, com base nos dados da POF 2008-2009. Apenas 7\% dos domicílios investigados reportaram algum gasto com assistência odontológica e 14,5\% comprometeram $20 \%$ ou mais de sua renda com tal tipo de despesa. Os dispêndios positivos foram cerca de sete vezes maior entre os domicílios do quinto mais rico da população em comparação ao mais pobre. Embora os domicílios mais ricos desembolsem um valor maior com serviços odontológicos, o comprometimento na renda dos domicílios do quinto mais pobre chega a ser dez vezes maior em relação aos mais ricos.

De acordo com uma análise da Pesquisa Mundial de Saúde 2002-2004, conduzida em 41 países de baixa e média renda, o percentual global de domicílios com gastos catastróficos com assistência odontológica no último mês, entre aqueles com gasto positivo, variou de 2,8\%, na Suazilândia, a 35\%, na Ucrânia, tendendo a ser maior em países mais desenvolvidos 15. O Brasil ocupou posição intermediária na pesquisa, com 25,3\% dos domicílios acometidos 15 . Comparações diretas com os dados encontrados na POF, neste ou em estudos previamente publicados, devem ser interpretados com cautela tendo em vista as diferenças metodológicas quanto à coleta de informações e classificação dos gastos.

O padrão de despesas privadas com odontologia vem ao encontro de estudos realizados sobre gastos com serviços de saúde e compra de medicamentos 7,8,10,16. Valores elevados do indicador de 
Figura 2

Média de gastos domiciliares per capita positivos (em Reais) e proporção média da renda familiar comprometida com assistência odontológica de acordo com os estados brasileiros. Pesquisa de Orçamentos Familiares (POF), Brasil, 2008-2009.

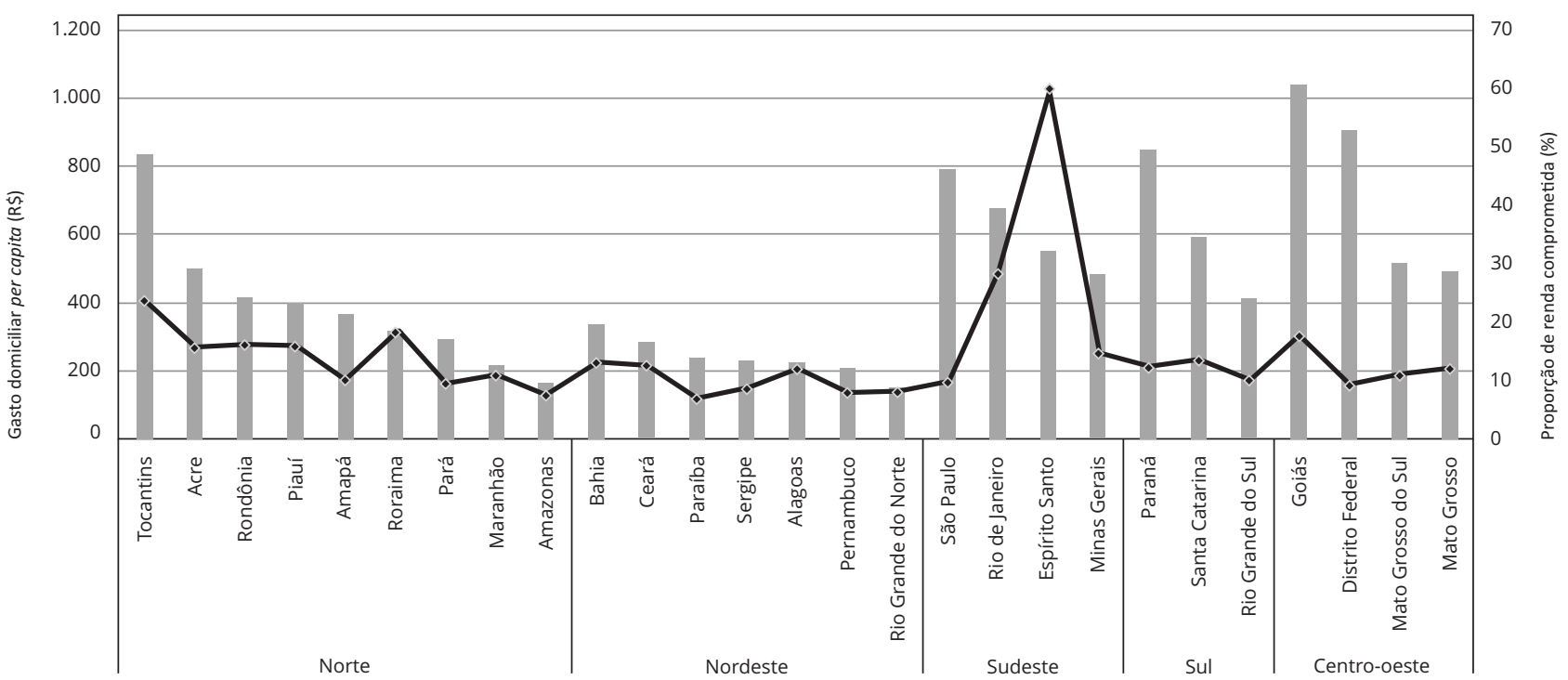

Gasto médio per capita (R\$)

- Comprometimento de renda (proporção média)

proporção da renda comprometida com saúde podem estar relacionados a baixos níveis de desenvolvimento socioeconômico e à insuficiência de serviços públicos 14 . Além desses, fatores como percepção de necessidade de tratamento, questões psicossociais, seguridade social, contexto epidemiológico e sociocultural também são determinantes importantes da compra de serviços de saúde 17 e poderiam ser explorados em futuras pesquisas.

O fato de o gasto absoluto com assistência odontológica ser superior entre os mais ricos sugere uma maior disponibilidade de recursos financeiros ou o pagamento de serviços mais caros, como os procedimentos odontológicos especializados (implantes, próteses e aparelhos ortodônticos), que contabilizam cerca de $85 \%$ das despesas referidas com odontologia 9 . Além disso, indivíduos com renda mais elevada optam pelo pagamento direto de serviços odontológicos privados a ter que arcar com algumas desvantagens provenientes da utilização dos serviços do setor público, tais como filas de espera, ou então adquirem planos odontológicos que objetivam financiar ou até mesmo reduzir os custos de seus tratamentos 18 .

A introdução da Política Nacional de Saúde Bucal, em 2004, impulsionou a expansão dos serviços de atenção primária, Centros de Especialidades Odontológicas, Laboratórios Regionais de Próteses Dentárias e fortaleceu a recomendação sobre fluoretação das águas de abastecimento público 19. Algumas melhorias nas condições de saúde bucal da população brasileira registradas, particularmente na faixa etária de adolescentes e adultos jovens, têm sido atribuídas à expansão dos serviços públicos odontológicos e de políticas de saúde do país. A possível cobertura mais abrangente desses serviços pode ter contribuído com o reduzido valor absoluto gasto nas camadas mais pobres, como também explicar o baixo percentual de domicílios que reportaram despender com assistência odontológica, de um modo geral. Esta hipótese vem ao encontro dos achados de Garcia et al. ${ }^{6}$ que demonstraram redução dos valores globais com gasto privado com serviços odontológicos ao longo das três últimas POF e, o trabalho de Boing et al. ${ }^{5}$ que identificou uma redução do percentual de famílias que se tornaram mais pobres por causa dos gastos com assistência odontológica. Em contrapartida, outras hipóteses 
que podem explicar o menor gasto com assistência odontológica entre as famílias mais pobres devem ser discutidas. Por exemplo, a oferta de serviços odontológicos privados é menor em locais próximos a comunidades de baixa renda. Outra possibilidade é o fato de as necessidades odontológicas não estarem entre as prioridades de consumo nas famílias de baixa renda, restringindo a compra por esses serviços.

Os dados devem ser interpretados com menos otimismo quando analisamos as desigualdades destacadas, uma vez que o impacto na renda das famílias menos favorecidas socioeconomicamente é quase dez vezes maior. Com relação ao acesso e utilização dos serviços odontológicos no Brasil, evidências apontam que, apesar dos avanços, as iniquidades permanecem, desfavorecendo mulheres, indivíduos mais jovens, menos escolarizados e pardos ${ }^{12}$. O subfinanciamento crônico do setor público de saúde no Brasil, quando comparado a outros países, compromete a capacidade de o sistema cumprir, com qualidade, os seus princípios de universalidade, integralidade e equidade. A restrição financeira agrava ainda mais os problemas de gestão do sistema, especialmente em regiões acometidas por problemas sociais, como a pobreza, desigualdade e baixo nível de educação e condições sanitárias 20,21. A continuidade e expansão da Política Nacional de Saúde Bucal e a garantia do acesso aos serviços odontológicos aos grupos menos favorecidos é essencial para que, em longo prazo, sejam percebidas a diminuição da participação dos gastos privados por parte dessas famílias e a redução das desigualdades apontadas.

Ao examinar variações geográficas do comprometimento da renda familiar com saúde, situações de desigualdade são igualmente identificadas. As regiões que mais gastam (Centro-oeste, Sudeste e Sul) são também as mais ricas do país, com maior acesso e utilização de serviços odontológicos 22,23 e as que apresentam um maior percentual de domicílios que comprometem sua renda com assistência odontológica. Esse dado ressalta as disparidades socioeconômicas e de acesso a serviços que tendem a ser maiores também nessas regiões em relação ao Norte e Nordeste. Em todos os quintos de renda, foram os domicílios da zona rural que apresentaram maior comprometimento no orçamento se comparados aos da zona urbana. Tal achado pode refletir melhor qualidade e abrangência dos serviços públicos em locais urbanizados 24 , o que poderia reduzir os gastos com tratamentos, ou até mesmo uma maior oferta de serviços privados odontológicos que, por conta da concorrência, oferecem opções de compra de tratamentos de menor custo.

As categorias de análise do percentual de renda comprometida foram selecionadas para fins de comparação com outros estudos e avaliação ao longo do tempo por serem amplamente utilizadas, não por representarem uma medida objetiva do que é um comprometimento alto ou baixo. O fato de analisar o período de um ano, aliado ao recordatório de 90 dias utilizado pela POF, não significa que essas despesas sejam mantidas ao longo do tempo, ocasionando um impacto persistente na renda. $O$ estudo não distingue famílias que tiveram um episódio de altos gastos daquelas com despesas contínuas e que porventura possam não ter sido incluídas na categoria de percentual mais alto de comprometimento na renda. Em se tratando de odontologia, a primeira situação é a mais provável, ou seja, os gastos tendem a ser altos e episódicos. Por um lado, o recordatório de noventa dias reduz o viés de informação, por outro, reduz a possibilidade de captar os gastos domiciliares episódicos ao longo do ano. Devido ao enorme número de domicílios com gasto nulo, optou-se por investigar o comprometimento na renda apenas aqueles que referiram algum gasto, de forma a produzir estimativas mais precisas. Essa análise acaba por limitar o universo amostral, não sendo possível inferir os resultados para a população como um todo e sim somente para aqueles que gastam. Outra limitação, originária da pesquisa, é que as despesas e os rendimentos são autorreferidos, o que pode não representar de forma fidedigna os reais valores despendidos. Por fim, os resultados apresentados refletem a situação dos gastos no período de 2008 a 2009. Tendo em vista mudanças observadas após esse período, tanto em termos de expansão de cobertura de serviços do SUS, como de crise econômica, com impacto na renda das famílias, é possível que a situação hoje seja diferente.

Apesar das limitações listadas, a utilização dos dados provenientes da POF é de suma importância. É uma pesquisa bem planejada e executada por meio de coleta primária de dados e instrumentos revisados, gerando uma fonte de dados valiosa para o estudo dos gastos das famílias brasileiras. Não identificamos na literatura outro estudo de igual especificidade, destacando o ineditismo deste trabalho.

Este estudo fornece elementos que permitem uma melhor compreensão das desigualdades no comprometimento do orçamento das famílias brasileiras com assistência odontológica e comple- 
menta as informações já publicadas. A continuidade e a expansão da Política Nacional de Saúde Bucal, bem como a elaboração de propostas que contribuam com a redução das desigualdades apontadas e garantam acesso a serviços odontológicos aos estratos socioeconomicamente menos favorecidos da população brasileira devem ser reforçadas.

\section{Colaboradores}

A. M. Cascaes concebeu o estudo, realizou análise e interpretação dos dados, redigiu o manuscrito e realizou a revisão crítica. A. M. Menegaz e A. R. Spohr auxiliaram na redação, interpretação os dados e realizaram a revisão crítica do manuscrito. R. A. Bomfim auxiliou na interpretação e discussão dos dados, realizou a revisão crítica do manuscrito. A. J. D. Barros participou na análise dos dados e realizou a revisão crítica do manuscrito.

\section{Agradecimentos}

Ao Conselho Nacional de Desenvolvimento Científico e Tecnológico (CNPq; processo número 447320/2014-9) pelo apoio financeiro para realização da análise dos dados.

\section{Referências}

1. Organização Mundial da Saúde. Relatório Mundial de Saúde 2010. Financiamento dos sistemas de saúde: o caminho para a cobertura universal. Genebra: Organização Mundial da Saúde; 2010.

2. World Health Organazation. WHO global health expenditure atlas. Geneva: World Health Organazation; 2012.

3. Instituto Brasileiro de Geografia e Estatística. Conta-satélite de saúde: Brasil 2010-2013. Rio de Janeiro: Instituto Brasileiro de Geografia e Estatística; 2015.

4. van Doorslaer E, O'Donnell O, Rannan-Eliya RP, Somanathan A, Adhikari SR, Garg CC, et al. Effect of payments for health care on poverty estimates in 11 countries in Asia: an analysis of household survey data. Lancet 2006; 368:1357-64.

5. Boing AC, Bertoldi AD, Posenato LG, Peres KG. The influence of health expenditures on household impoverishment in Brazil. Rev Saúde Pública 2014; 48:797-807.

6. Garcia LP, Sant'Anna AC, Magalhães LCG, Aurea PA. Gastos com saúde das famílias brasileiras residentes em regiões metropolitanas: composição e evolução no período 1995-2009. Ciênc Saúde Coletiva 2013; 18:115-28.

7. Garcia LP, Sant'Anna AC, Magalhães LCG, Freitas LRS, Aurea AP. Gastos das famílias brasileiras com medicamentos segundo a renda familiar: análise da Pesquisa de Orçamentos Familiares de 2002-2003 e de 2008-2009. Cad Saúde Pública 2013; 29:1605-16.

8. Garcia LP, Ocké-Reis CO, Magalhães LCG, Sant'Anna AC, Freitas LRS. Gastos com planos de saúde das famílias brasileiras: estudo descritivo com dados das Pesquisas de Orçamentos Familiares 2002-2003 e 2008-2009. Ciênc Saúde Coletiva 2015; 20:1425-34.

9. Cascaes AM, Camargo MBJ, Castilhos ED, Silva AER, Barros AJ. Gastos privados com saúde bucal no Brasil: análise dos dados da Pesquisa de Orçamentos Familiares, 2008-2009. Cad Saúde Pública 2017; 33:e00148915.

10. Boing AC, Bertoldi AD, Barros AJD, Posenato LG, Peres KG. Socioeconomic inequality in catastrophic health expenditure in Brazil. Rev Saúde Pública 2014; 48:632-41. 
11. Barros AJD, Bastos JL, Dâmaso AH. Catastrophic spending on health care in Brazil: private health insurance does not seem to be the solution. Cad Saúde Pública 2011; 27 Suppl 2:S254-62.

12. Peres MA, Iser BP, Boing AF, Yokota RT, Malta DC, Peres KG. Desigualdades no acesso e na utilização de serviços odontológicos no Brasil: analise do Sistema de Vigilância de Fatores de Risco e Proteção para Doenças Crônicas por Inquérito Telefônico (VIGITEL 2009). Cad Saúde Pública 2012; 28 Suppl:S90-100.

13. Coordenação Geral de Saúde Bucal, Departamento de Atenção Básica, Secretaria de Atenção à Saúde, Secretaria de Vigilância em Saúde, Ministério da Saúde. Projeto SBBrasil 2010: Pesquisa Nacional de Saúde Bucal. Resultados principais. Brasília: Ministério da Saúde; 2011.

14. Instituto Brasileiro de Geografia e Estatística. Pesquisa de Orçamentos Familiares 20082009: perfil das despesas no Brasil. Indicadores selecionados. Brasília: Instituto Brasileiro de Geografia e Estatística; 2012.

15. Masood M, Sheiham A, Bernabe E. Household expenditure for dental care in low and middle income countries. PLoS One 2015; 10:e0123075.

16. Boing AC, Bertoldi AD, Peres KG. Socioeconomic inequalities in expenditures and income committed to the purchase of medicines in Southern Brazil. Rev Saúde Pública 2011; 45:897-905.
17. Zucchi P, Del Nero C, Malik A. Gastos em saúde: os fatores que agem na demanda e na oferta dos serviços de saúde. Saúde Soc 2000; 9:12750.

18. Agência Nacional de Saúde Suplementar. Planos odontológicos: uma abordagem econômica no contexto regulatório. Rio de Janeiro: Agência Nacional de Saúde Suplementar; 2002.

19. Aquilante AG, Aciole GG. Oral health care after the National Policy on Oral Health - "Smiling Brazil": a case study. Ciênc Saúde Coletiva 2015; 20:239-48.

20. Ocké-Reis CO. O mercado de planos de saúde: o problema vira solução? Ciênc Saúde Coletiva 2008; 13:1398-400.

21. Ocké-Reis CO. Gasto privado em saúde no Brasil. Cad Saúde Pública 2015; 31:1351-3.

22. Martins RC, Reis CM, Machado ATM, Amaral JH, Werneck MA, Abreu MH. Relationship between primary and secondary dental care in public health services in Brazil. PLoS One 2016; 11:e0164986.

23. Viacava F, Bellido JG. Health, access to services and sources of payment, according to household surveys. Ciênc Saúde Coletiva 2016; 21:351-70.

24. Kassouf AL. Acesso aos serviços de saúde nas áreas urbana e rural do Brasil. Revista de Economia e Sociologia Rural 2005; 43:29-44. 


\section{Abstract}

The study aimed to investigate inequalities in the commitment of family income to private expenditures on dental care in Brazil. Data were analyzed from 55,970 Brazilian households that participated in the nationwide Family Budgets Survey in 2008-2009. The commitment of family income to private spending on dental care was calculated by dividing the mean annual per capita household spending on dental care by the mean annual per capita income, classified in four categories: $>0 \%$, $\geq 5 \%, \geq 10 \%$, and $\geq 20 \%$. Analysis of income commitment only included households with positive spending. Only 2,961 households (7\%) reported positive spending on dental care. Mean annual per capita spending was BRL 42.19 (USD 12.78) overall and BRL 602.47 (USD 182.57) among those with positive spending. Households with the highest absolute expenditures on dental care were those from urban areas and the wealthiest quintile. Meanwhile, households with the highest proportional income commitment were from rural areas and the poorest quintile. Among those that reported positive spending, 55\% of the households in the poorest quintile committed $\geq 20 \%$ of their income to dental care. The proportion was only $6 \%$ in the wealthiest quintile of the population. The poorest households in the wealthiest regions of Brazil (Central, South, and Southeast) showed the highest income commitments. There were striking socioeconomic inequalities in spending and income commitment to dental care. The evaluation of these inequalities is relevant for the evaluation and orientation of public health policies.

Dental Economics; Income; Health Expenditures

\section{Resumen}

El objetivo de este trabajo fue investigar las desigualdades en el desembolso de renta domiciliaria con gastos privados en asistencia odontológica en Brasil. Se analizaron datos de 55.970 domicilios brasileños que participaron en la Encuesta de Presupuestos Familiares, de alcance nacional, durante el período de 2008-2009. El desembolso económico privado en asistencia odontológica, respecto a la renta familiar, se calculó mediante la división del gasto medio domiciliario per cápita anual por la renta familiar per cápita anual y estimado según cuatro categorías: $>0 \%, \geq 5 \%, \geq$ $10 \% y \geq 20 \%$. El análisis del desembolso económico se realizó sólo para los domicilios con gasto positivo. Sólo 2.961 (7\%) de los domicilios informaron de gastos positivos con asistencia en odontología. En general, el gasto medio per cápita anual fue BRL 42,19y, BRL 602,47 entre quienes contaban con un gasto positivo. Los domicilios que cuentan con los mayores gastos absolutos respecto a la asistencia odontológica son los pertenecientes al área urbana y a la quinta parte más rica. En contrapartida, los domicilios con mayor desembolso de renta pertenecen al área rural y a la quinta parte más pobre. Entre los que informaron de gasto positivo, un $55 \%$ de los domicilios de la quinta parte más pobre desembolsaron un $\geq 20 \%$ de su renta en asistencia odontológica. El porcentaje es de apenas un $6 \%$ en el grupo más rico de la población. Los domicilios más pobres de las regiones más ricas (Centro-oeste, Sur y Sudeste) presentaron los mayores desembolsos de renta. Las desigualdades socioeconómicas en los gastos y en el desembolso de renta con la asistencia odontológica son evidentes. La evaluación de estas desigualdades es relevante para la evaluación y orientación de las políticas públicas en salud.

Economía em Odontología; Renta; Gastos en Salud
Recebido em 17/Jun/2017

Versão final reapresentada em 25/Dez/2017

Aprovado em 26/Jan/2018 